\title{
A Variationally Computed IR Line List for the Methyl Radical $\mathrm{CH}_{3}$
}

\author{
Ahmad Y. Adam, ${ }^{\dagger}$ Andrey Yachmenev, ${ }^{\ddagger}$ Sergei N. Yurchenko, $₫$ and Per Jensen ${ }^{*, \dagger}$ \\ $\dagger$ Fakultät für Mathematik und Naturwissenschaften, Physikalische und Theoretische \\ Chemie, Bergische Universität Wuppertal, D-42097 Wuppertal, Germany \\ $\ddagger$ Center for Free-Electron Laser Science, Deutsches Elektronen-Synchrotron DESY, \\ Notkestraße 85, D-22607 Hamburg, Germany, The Hamburg Center for Ultrafast Imaging, \\ Universität Hamburg, Luruper Chaussee 149, D-22761 Hamburg, Germany \\ 【Department of Physics and Astronomy, University College London, Gower Street, London \\ WC1E 6BT, United Kingdom \\ E-mail: jensen@uni-wuppertal.de
}




\begin{abstract}
We present the first variational calculation of a hot temperature ab initio line list for the $\mathrm{CH}_{3}$ radical. It is based on a high level ab initio potential energy surface and dipole moment surface of $\mathrm{CH}_{3}$ in the ground electronic state. The ro-vibrational energy levels and Einstein $A$ coefficients were calculated using the general-molecule variational approach implemented in the computer program TROVE. Vibrational energies and vibrational intensities are found to be in very good agreement with the available experimental data. The line list comprises $9,127,123$ ro-vibrational states $(J \leq 40)$ and 2,058,655,166 transitions covering the wavenumber range up to $10000 \mathrm{~cm}^{-1}$ and should be suitable for temperatures up to $T=1500 \mathrm{~K}$.
\end{abstract}

\title{
Introduction
}

The methyl radical $\mathrm{CH}_{3}$ is a free radical of major importance in many areas of science such as hydrocarbon combustion processes, ${ }^{1}$ atmospheric chemistry, ${ }^{2}$ the chemistry of semiconductor production, ${ }^{3}$ the chemical vapor deposition of diamond, ${ }^{4}$ and many chemical processes of current industrial and environmental interest. It is also expected to be present in exoplanetary atmospheres,${ }^{5}$ in the atmospheres of Saturn ${ }^{6}$ and Neptune,${ }^{7}$ and in the interstellar medium, ${ }^{8}$ where it is thought to be one of the most abundant free radicals. ${ }^{5}$ Because of its central role in this variety of situations, its structural and spectroscopic parameters have been extensively studied. Diverse spectroscopic techniques have been employed to determine absolute concentrations of $\mathrm{CH}_{3}$ in the gas phase, including $\mathrm{UV} /$ visible, ${ }^{9}$ infrared, ${ }^{10}$ and Raman spectroscopies. ${ }^{11-17}$ In addition, $\mathrm{CH}_{3}$ is an example of a molecule with large vibrational contribution to the hyperfine coupling constant, accounting for up to about $41 \%$ of the total value (see Ref. 18 and references therein).

Owing to the importance of $\mathrm{CH}_{3}$ in various contexts, in particular in astrophysics and -chemistry, its concentrations or column densities in remote environments such as interstellar space, the terrestrial atmosphere, exo-planetary atmospheres, and the outer layers of cool 
stars are of interest and it is desirable to determine these by remote-sensing spectroscopic methods. A prerequisite for such determinations is the knowledge of the transition moments for the observed transitions, and these must often be obtained in theoretical calculations as done, for example, in the ExoMol project ${ }^{19,20}$ by Yurchenko and co-workers. ${ }^{21-33}$ This project aims at providing theoretically computed transition moments and simulated spectra for (small to medium-sized) general polyatomic molecules of astrophysical and/or -chemical interest. In general, molecular transition moments known to within 10-20\% (the typical accuracy of the transition moment data of the HITRAN database ${ }^{34}$ ) are sufficiently accurate to be useful for most applications involving determination of concentrations and column densities, at least for fundamental and overtone bands. The level of ab initio theory used in the present study [RCCSD(T)-F12b /cc-pVQZ-F12; see below] is expected to be sufficient to satisfy this requirement for the transition moments computed in the present work. Also, we expect the inaccuracy of our calculations to be predominantly caused by the inaccuracy of the ab initio potential energy surface, and not by the truncation of the kinetic energy operator expansion.

At equilibrium, the three protons of electronic-ground-state $\tilde{X}^{2} A_{2}^{\prime \prime} \mathrm{CH}_{3}$ form an equilateral triangle with the $\mathrm{C}$ nucleus at the centre-of-mass of the planar structure with $\boldsymbol{D}_{3 \mathrm{~h}}$ point group symmetry (see Table A-10 of Ref. 35). There is no permanent dipole moment, and so the pure rotational transitions are dipole forbidden and very weak. Also, the planar ground-state equilibrium structure precludes most one-photon transitions to excited electronic states. ${ }^{36}$ Owing to the extremely weak rotational spectrum, determinations of concentrations and column densities for $\mathrm{CH}_{3}$ must be made with rovibrational transitions in the infrared region. The most suitable transitions are those in the intense $\nu_{2}$ fundamental band at $606 \mathrm{~cm}^{-1}$ (where $\nu_{2}$ is the out-of-plane bending mode). This band provides convenient transitions for concentration measurements and has been used extensively for this purpose. ${ }^{37-40}$ As mentioned above, the corresponding transition moments must be known in order that concentrations can be determined, and the present work can be viewed as a 
first step towards providing extensive catalogues of theoretical transition moments for $\mathrm{CH}_{3}$, so-called line lists, of use in astrophysical studies. In the present work we apply a high level ab initio potential energy surface, refined by means of experimental spectroscopic data, and $a b$ initio dipole moment surfaces to compute, with the TROVE program, ${ }^{41-44}$ sufficient energies and transition moments for generating a hot $(T=1500 \mathrm{~K})$ IR line list for $\mathrm{CH}_{3}$.

\section{Theory}

\section{Potential energy surface}

The potential energy surface (PES) employed for the electronic ground state of $\mathrm{CH}_{3}$ in the present work is based on the ab initio surface reported in Ref. 18, which we denote as PES-1. The PES-1 electronic energies were computed for 24000 symmetry-unique molecular geometries at the open-shell RCCSD(T)-F12b $\mathrm{b}^{45,46}$ level of theory (explicitly correlated F12 restricted coupled cluster included single and double excitations with a noniterative correction for triples) and the F12-optimized correlation consistent polarized valence basis set cc-pVQZ-F12. ${ }^{47}$ The carbon inner-shell electron pair was treated as frozen core in the correlated calculations. By using the frozen-core approximation we benefit from error cancellation. It is well known that, e.g. for second-row atoms, the core-valence correlation is almost exactly cancelled by the more costly high-order correlation effects. ${ }^{48}$ Keeping only one of them would make the accuracy deteriorate.

The analytical representation for the PES was obtained in a least-squares fitting procedure using the parameterized function from Lin et al. ${ }^{49}$ : 


$$
\begin{aligned}
V\left(\xi_{1}, \xi_{2}, \xi_{3}, \xi_{4 a}, \xi_{4 b} ; \sin \bar{\rho}\right) & =V_{\mathrm{e}}+V_{0}(\sin \bar{\rho})+\sum_{j} F_{j}(\sin \bar{\rho}) \xi_{j} \\
& +\sum_{j \leqslant k} F_{j k}(\sin \bar{\rho}) \xi_{j} \xi_{k}+\sum_{j \leqslant k \leqslant l} F_{j k l}(\sin \bar{\rho}) \xi_{j} \xi_{k} \xi_{l} \\
& +\sum_{j \leqslant k \leqslant l \leqslant m} F_{j k l m}(\sin \bar{\rho}) \xi_{j} \xi_{k} \xi_{l} \xi_{m}+\ldots
\end{aligned}
$$

this function depends on the stretching variables

$$
\xi_{k}=1-\exp \left[-a\left(r_{k}-r_{\mathrm{e}}\right)\right], \quad k=1,2,3,
$$

where $r_{k}$ is the instantaneous value of the distance between the $\mathrm{C}$ nucleus and the proton $\mathrm{H}_{k}$ labeled $k=1,2$, or $3 ; r_{\mathrm{e}}$ is the common equilibrium value of the three $r_{k}$ bond lengths, and $a$ is a Morse parameter. Furthermore, the symmetrized bending variables $\left(\xi_{4 a}, \xi_{4 b}\right)$ are defined as

$$
\left(\xi_{4 a}, \xi_{4 b}\right)=\left(\frac{1}{\sqrt{6}}\left[2 \alpha_{1}-\alpha_{2}-\alpha_{3}\right], \frac{1}{\sqrt{2}}\left[\alpha_{2}-\alpha_{3}\right]\right)
$$

with $\alpha_{i}$ as the bond angle $\angle\left(\mathrm{H}_{j} \mathrm{XH}_{k}\right)$ where $(i, j, k)$ is a permutation of the numbers $(1,2,3)$. Finally, the variable

$$
\sin \bar{\rho}=\frac{2}{\sqrt{3}} \sin \left[\left(\alpha_{1}+\alpha_{2}+\alpha_{3}\right) / 6\right]
$$

describes the out-of-plane bending. At the planar equilibrium configuration, we have $\alpha_{1}+$ $\alpha_{2}+\alpha_{3}=360^{\circ}$ and $\operatorname{so} \sin \bar{\rho}=\sin \bar{\rho}_{\mathrm{e}}=1$. The functions $V_{0}(\sin \bar{\rho})$ and $F_{j k \ldots}(\sin \bar{\rho})$ in Eq. (1) are defined as

$$
\begin{aligned}
V_{0}(\sin \bar{\rho}) & =\sum_{s=1}^{4} f_{0}^{(s)}(1-\sin \bar{\rho})^{s}, \\
F_{j k \ldots}(\sin \bar{\rho}) & =\sum_{s=0}^{N} f_{j k \ldots}^{(s)}(1-\sin \bar{\rho})^{s},
\end{aligned}
$$


where the quantities $f_{0}^{(s)}$ and $f_{j k \ldots}^{(s)}$ in Eqs. (5) and (6) are expansion coefficients. The optimized values of the parameters $a, r_{\mathrm{e}}, f_{0}^{(s)}$, and $f_{j k \ldots}^{(s)}$ are given in the supplementary material to Ref. 18 together with Fortran 90 routine for calculating PES values.

The analytical form of PES-1 ${ }^{18}$ is given in terms of the $a b$ initio cc-pVQZ-F12 values of the equilibrium structural parameters, $r_{\mathrm{e}}=1.0774 \AA$ and $\alpha_{\mathrm{e}}=120^{\circ}{ }^{18}$ for the electronic ground state of $\mathrm{CH}_{3}$. In the present work, we optimized the value of $r_{\mathrm{e}}$ in a least-squares fitting to experimentally derived to rotational energy spacings within the vibrational states of $\mathrm{CH}_{3}$. The fitting produced $r_{\mathrm{e}}=1.0763 \AA ; \alpha_{\mathrm{e}}=120^{\circ}$ by symmetry. We use these optimized values of the equilibrium structural parameters. All results presented below are based on the analytical potential energy function called PES-2, obtained from PES-1 ${ }^{18}$ by replacing the ab initio cc-pVQZ-F12 value of $r_{\mathrm{e}}$ by the adjusted value given here. The remaining PES-2 parameter values are identical to those of PES-1 and can be obtained from the supplementary material to Ref. 18.

\section{Dipole moment surface}

The dipole moment surface (DMS) for the electronic ground state of $\mathrm{CH}_{3}$ was computed using the MOLPRO ${ }^{50}$ program package. Frozen-core calculations were carried out for 19361 symmetry-unique geometries (15 600 below $30000 \mathrm{~cm}^{-1}$ ) using the spin-restricted open-shell coupled cluster theory $\operatorname{RCCSD}(\mathrm{T})^{51}$ and the augmented correlation consistent valence basis set aug-cc-pVTZ, ${ }^{52,53}$ employing the two-point stencil central finite differences with the electric field strength of 0.002 a.u.

We employ the so-called symmetrized molecular-bond (SMB) representation ${ }^{22}$ [which is an extension of the molecular-bond (MB) representation ${ }^{54}$ ] to formulate analytical functions describing the molecular dipole moment components. The SMB representation makes use of the projections $\overline{\boldsymbol{\mu}} \cdot \mathbf{e}_{k}$ of the dipole moment on the molecular bonds, where $\mathbf{e}_{k}$ is the unit vector along the $\mathrm{C}-\mathrm{H}_{k}$ bond,

$$
\mathbf{e}_{k}=\frac{\mathbf{r}_{k}-\mathbf{r}_{4}}{\left|\mathbf{r}_{k}-\mathbf{r}_{4}\right|}
$$


with $\mathbf{r}_{k}, k=1,2,3$, as the position vector of proton $k$ and $\mathbf{r}_{4}$ as the position vector of the C nucleus.

We form symmetry-adapted linear combinations of the MB projections $\overline{\boldsymbol{\mu}} \cdot \mathbf{e}_{j}$ :

$$
\begin{aligned}
\bar{\mu}_{A_{2}^{\prime \prime}}^{\mathrm{SMB}} & =\left(\overline{\boldsymbol{\mu}} \cdot \mathbf{e}_{\mathrm{N}}\right) \\
\bar{\mu}_{E_{a}^{\prime}}^{\mathrm{SMB}} & =\frac{1}{\sqrt{6}}\left[2\left(\overline{\boldsymbol{\mu}} \cdot \mathbf{e}_{1}\right)-\left(\overline{\boldsymbol{\mu}} \cdot \mathbf{e}_{2}\right)-\left(\overline{\boldsymbol{\mu}} \cdot \mathbf{e}_{3}\right)\right] \\
\bar{\mu}_{E_{b}^{\prime}}^{\mathrm{SMB}} & =\frac{1}{\sqrt{2}}\left[\left(\overline{\boldsymbol{\mu}} \cdot \mathbf{e}_{2}\right)-\left(\overline{\boldsymbol{\mu}} \cdot \mathbf{e}_{3}\right)\right],
\end{aligned}
$$

where, in addition to the vectors $\mathbf{e}_{k}$, we have introduced $\mathbf{e}_{\mathrm{N}}=\mathbf{q}_{\mathrm{N}} /\left|\mathbf{q}_{\mathrm{N}}\right|$ with $\mathbf{q}_{\mathrm{N}}$ as the 'trisector'

$$
\mathbf{q}_{\mathrm{N}}=\left(\mathbf{e}_{1} \times \mathbf{e}_{2}\right)+\left(\mathbf{e}_{2} \times \mathbf{e}_{3}\right)+\left(\mathbf{e}_{3} \times \mathbf{e}_{1}\right) .
$$

The subscripts $\Gamma=A_{2}^{\prime \prime}, E_{a}^{\prime}$, and $E_{b}^{\prime}$ of the quantities $\bar{\mu}_{\Gamma}^{\mathrm{SMB}}$ in Eqs. (8)-(10) refer to the irreducible representations (Table A-10 of Ref. 35) of the $\mathrm{CH}_{3}$ molecular symmetry group $\boldsymbol{D}_{3 \mathrm{~h}}(\mathrm{M})$; the electronically averaged dipole moment $\overline{\boldsymbol{\mu}}$ generates the representation $A_{2}^{\prime \prime} \oplus$ $E^{\prime}$. The quantity $\bar{\mu}_{A_{2}^{\prime \prime}}^{\mathrm{SMB}}$ is antisymmetric under the inversion operation ${ }^{35} E^{*}$ and vanishes at planarity, so that $\overline{\boldsymbol{\mu}}$ has only two non-vanishing, linearly independent components at planarity. These two components vanish at planar configurations with $\boldsymbol{D}_{3 \mathrm{~h}}$ point group symmetry.

The three components of the SMB dipole moment in Eqs. (8)-(10) are represented by $4^{\text {th }}$ order polynomial expansions

$$
\begin{gathered}
\bar{\mu}_{\Gamma}^{\mathrm{SMB}}\left(\chi_{1}, \chi_{2}, \chi_{3}, \chi_{4 a}, \chi_{4 b} ; \rho\right)=\mu_{0}^{\Gamma}(\sin \bar{\rho})+\sum_{i} \mu_{i}^{\Gamma}(\sin \bar{\rho}) \chi_{i}+\sum_{i \leq j} \mu_{i j}^{\Gamma}(\sin \bar{\rho}) \chi_{i} \chi_{j} \\
+\sum_{i \leq j \leq k} \mu_{i j k}^{\Gamma}(\sin \bar{\rho}) \chi_{i} \chi_{j} \chi_{k}+\sum_{i \leq j \leq k \leq l} \mu_{i j k l}^{\Gamma}(\sin \bar{\rho}) \chi_{i} \chi_{j} \chi_{k} \chi_{l},
\end{gathered}
$$

in terms of the variables

$$
\chi_{k}=\Delta r_{k}\left(1-\exp \left(-\Delta r_{k}\right)\right)^{2}, \quad(k=1,2,3)
$$


with $\Delta r_{k}=r_{k}-r_{\mathrm{e}}$ and

$$
\left(\chi_{4}, \chi_{5}\right)=\left(\xi_{4 a}, \xi_{4 b}\right)
$$

where $\left(\xi_{4 a}, \xi_{4 b}\right)$ are defined in Eq. (3). The expansion coefficients $\mu_{i j \ldots}^{\Gamma}(\sin \bar{\rho})$ are defined as

$$
\mu_{i j \ldots}^{\Gamma}(\sin \bar{\rho})=\sum_{s=0}^{N} \mu_{i j \ldots}^{\Gamma(s)}(1-\sin \bar{\rho})^{s}
$$

where $\sin \bar{\rho}$ is given by Eq. (4) and the maximal order of the polynomial is $N=8$. For more details the reader is referred to Ref. 22. The final fit of the 15600 geometries required a total number of 218 parameters (131 for $\bar{\mu}_{A_{2}^{\prime \prime}}^{\mathrm{SMB}}$ and 87 for $\bar{\mu}_{E_{a}^{\prime}}^{\mathrm{SMB}}$ and $\bar{\mu}_{E_{b}^{\prime}}^{\mathrm{SMB}}$ ) and reproduced the ab initio data with a root-mean-square (RMS) differences of $0.003 \mathrm{D}, 0.001 \mathrm{D}$ and $0.004 \mathrm{D}$ for the $x, y$ and $z$ components respectively and energies up to $30000 \mathrm{~cm}^{-1}$, see Figure 1 . A series of fittings to the $x, y$ and $z a b$ initio dipole-moment components have been carried out, including in the data set for each fitting the ab initio points with electronic energy (relative to the potential energy minimum) $V \leqslant E$, and increasing $E$. Figure 1 shows the RMS deviation for each dipole-moment component as a function of $E$. The DMS expansion parameter set and the Fortran 90 functions are included in the supplementary material.

\section{Intensity simulations with TROVE}

The 'readiness' of the molecule to make an absorption or emission transition from an initial ro-vibrational state $i$ to a final ro-vibrational state $f$ is expressed by the line strength $35,55,56$ $S(f \leftarrow i)$, a quantity with units of [dipole moment] ${ }^{2}$ (typically Debye ${ }^{2}$. For $S(f \leftarrow i)=$ 0, the transition does not take place and need not be considered. As discussed in Ref. 35 transitions with $S(f \leftarrow i) \neq 0$ are said to satisfy selection rules which we can derive from symmetry considerations before we do quantitative, numerical calculations of $S(f \leftarrow i)$. Thus, these calculations need only be done for transitions satisfying the selection rules, and after obtaining values of $S(f \leftarrow i)$ for these transitions, we can compute the corresponding Einstein coefficients and absorption intensities. 
Figure 1: Root-mean-square (RMS) errors of the fittings to the $a b$ initio dipole moment values. The results of a series of fittings are shown. In each fitting, the data set includes the $a b$ initio points with electronic energy (relative to the potential energy minimum) $V \leqslant E$ (see text).

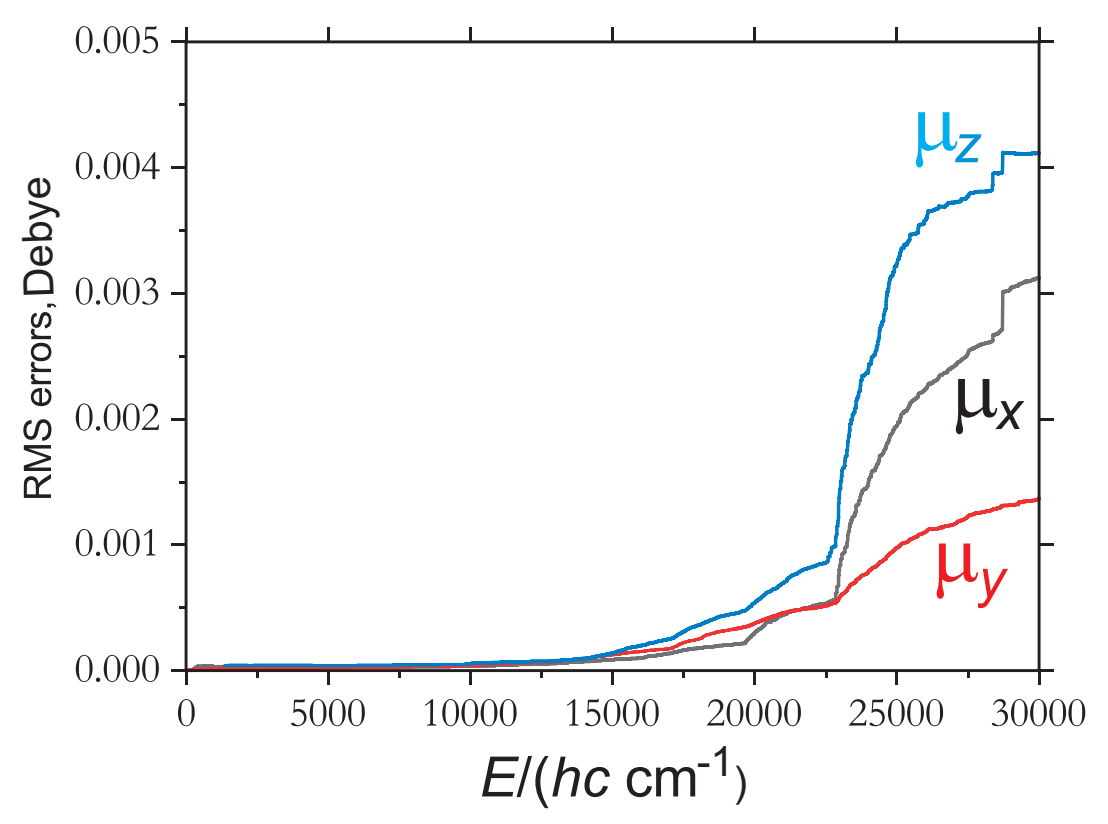

The initial(final) state $i(f)$ has the rotation-vibration wavefunction $\left|\Phi_{\mathrm{rv}}^{(i)}\right\rangle\left(\left|\Phi_{\mathrm{rv}}^{(f)}\right\rangle\right)$. The line strength ${ }^{35,55,56} S(f \leftarrow i)$ of the ro-vibrational transition $f \leftarrow i$ is

$$
S(f \leftarrow i)=g_{\mathrm{ns}} \sum_{M_{f}, M_{i}} \sum_{A=X, Y, Z}\left|\left\langle\Phi_{\mathrm{rv}}^{(f)}\left|\bar{\mu}_{A}\right| \Phi_{\mathrm{rv}}^{(i)}\right\rangle\right|^{2}
$$

where the nuclear spin statistical weight factor ${ }^{35}$ is denoted $g_{\text {ns }}$ and the electronically averaged component of the molecular dipole moment along the space-fixed axis ${ }^{35}$ is denoted $\bar{\mu}_{A}$, $A=X, Y$, or $Z$. The quantity $M_{i}\left(M_{f}\right)$ is the quantum number defining the projection of the total angular momentum $\hat{\mathbf{J}}$ on the $Z$ axis for the initial(final) state.

Assuming that the molecules considered are in thermal equilibrium at the absolute tem- 
perature $T$, the intensity of a spectral line is determined as

$$
I(f \leftarrow i)=\frac{8 \pi^{3} N_{A} \tilde{\nu}_{i f}}{\left(4 \pi \epsilon_{0}\right) 3 h c} \frac{\mathrm{e}^{-E_{i} / k T}}{Q}\left[1-\exp \left(-h c \tilde{\nu}_{i f} / k T\right)\right] S(f \leftarrow i) .
$$

Here, the absorption wavenumber is denoted $\tilde{\nu}$, and Eq. (17) yields the intensity of a transition from the initial state $i$ with energy $E_{i}$ to the final state $f$ with energy $E_{f}$, where $h c \tilde{\nu}_{i f}$ $=E_{f}-E_{i}$. The partition function $Q$ is defined as $Q=\sum_{j} g_{j} \exp \left(-E_{j} / k T\right)$, where $g_{j}$ is the total degeneracy of the state with energy $E_{j}$ and the sum runs over all energy levels of the molecule, and other symbols have their usual meanings. The total degeneracy $g_{j}$ is given by $(2 J+1)$ times the spin degeneracy $(2 S+1=2)$ and times the nuclear spin degeneracy which is $4,0,2,4,0,2$ for $A_{1}^{\prime}, A_{2}^{\prime}, E^{\prime}, A_{1}^{\prime \prime}, A_{2}^{\prime \prime}$, and $E^{\prime \prime}$ symmetries respectively. The ground electronic state of $\mathrm{CH}_{3}$ is a doublet $\left(\tilde{X}^{2} A_{2}^{\prime \prime}\right)$ with a small splitting ${ }^{57,58}$ in the rovibrational energy levels due to spin-rotation interactions, around $0.01 \mathrm{~cm}^{-1}$, which we therefore chose to ignore in the present work.

Yurchenko et al. ${ }^{55}$ have given, in their Eq. (21), a detailed expression for the line strength of an individual ro-vibrational transition within an isolated electronic state of an $\mathrm{XY}_{3}$ pyramidal molecule. Assuming that the populations of the lower (initial) states are Boltzmanndistributed, we limit the intentity calculations to transitions starting from levels below $E_{i}^{\max } / h c=9000 \mathrm{~cm}^{-1}$. With this limitation, Boltzmann factors of $\exp \left(-E_{i} / k T\right)>2 \times 10^{-4}$ enter into Eq. (17) for $T=1500 \mathrm{~K}$. It is common to use the partition function for estimating the completeness of the line list for a given temperature. ${ }^{24}$ Towards this end, we consider the ratio $Q_{9000 \mathrm{~cm}^{-1}} / Q_{\text {total }}$, where $Q_{\text {total }}$ is the converged partition function value calculated by explicit summation over all computed energy levels and $Q_{9000 \mathrm{~cm}^{-1}}$ is the partition function value calculated by summation over levels with energies lower than $9000 \mathrm{~cm}^{-1}$. This ratio gives $95 \%$ completeness at temperatures below $1500 \mathrm{~K}$. Consequently, we estimate $T=$ $1500 \mathrm{~K}$ to be the maximal temperature for which our line list is realistic. Since it is safe to limit the lower-state energies to be below $9000 \mathrm{~cm}^{-1}$, it is sufficient to consider rotational 
states with $J \leqslant 40$. We compute a line list in the wavenumber range $0-10000 \mathrm{~cm}^{-1}$; the upper energy limit (i.e., the maximum value of the final-state energy) corresponds to a term value of $E^{\max } / h c=19,000 \mathrm{~cm}^{-1}$.

\section{Computational details}

The variational nuclear-motion calculations are done with a symmetry-adapted basis set. With such a basis set, the Hamiltonian matrix becomes block diagonal according to the irreducible representations of the $\boldsymbol{D}_{3 \mathrm{~h}}(\mathrm{M})$ molecular symmetry group: ${ }^{35} A_{1}^{\prime}, A_{2}^{\prime}, A_{1}^{\prime \prime}, A_{2}^{\prime \prime}, E^{\prime}$, and $E^{\prime \prime}$. The $A_{2}^{\prime}$ and $A_{2}^{\prime \prime}$ matrices are of no interest for $\mathrm{CH}_{3}$ as the corresponding states have zero nuclear spin statistical weights and do not exist in nature. ${ }^{35}$ The $E^{\prime}$ and $E^{\prime \prime}$ matrices each split into two sub-blocks, of which only one must be diagonalized. ${ }^{35}$

The calculation of the matrix elements $\left\langle\Phi_{\mathrm{rv}}^{(f)}\left|\bar{\mu}_{A}\right| \Phi_{\mathrm{rv}}^{(i)}\right\rangle$ in Eq. (16) is the bottle-neck in the spectrum simulations. Here, the wavefunctions $\Phi_{\mathrm{rv}}^{(w)}$ are given as superpositions of symmetryadapted basis functions (see Eq. (65) of Yurchenko et al. ${ }^{44}$ ):

$$
\left|\Phi_{\mathrm{rv}}^{(w)}\right\rangle=\sum_{V K \tau_{\mathrm{rot}}} C_{V K \tau_{\mathrm{rot}}}^{(w)}\left|J_{w} K m_{w} \tau_{\mathrm{rot}}\right\rangle|V\rangle, w=i \text { or } f
$$

with the $C_{V K \tau_{\text {rot }}}^{(w)}$ as expansion coefficients. In Eq. (18), the symmetrized rotational basis functions are denoted $\left|J_{w} K m_{w} \tau_{\text {rot }}\right\rangle$ with $\tau_{\text {rot }}$ (=0 or 1$)$ defining the rotational parity, and $|V\rangle$ is a vibrational basis function. In order to accelerate this part of the calculation, we pre-screened the expansion coefficients $C_{V K \tau_{\text {rot }}}^{(f)}$. All terms with coefficients less than the threshold value of $10^{-13}$ were discarded in the intensity calculation.

The evaluation of the dipole moment matrix elements $\left\langle\Phi_{\mathrm{rv}}^{(f)}\left|\bar{\mu}_{A}\right| \Phi_{\mathrm{rv}}^{(i)}\right\rangle$ has been made more efficient in a two-step procedure. In the first step, an effective line strength is evaluated for a given lower state $i$ :

$$
S_{i, V K}^{A}=\left\langle\Phi_{\mathrm{rv}}^{(i)}\left|\bar{\mu}_{A}\right| \phi_{V K}\right\rangle
$$


Here, $\phi_{V K}$ is a short-hand notation for the primitive basis function $\left|J_{w} K m_{w} \tau_{\text {rot }}\right\rangle \times|V\rangle$. From the $S_{i, V K}$-values obtained, we compute, in the second step, the line strength $S(f \leftarrow i)$ as

$$
S(f \leftarrow i)=g_{\mathrm{ns}} \sum_{m_{i}, m_{f}} \sum_{A=X, Y, Z}\left|\sum_{V, K} C_{V K \tau_{\mathrm{rot}}}^{(f)} S_{i, V K}^{A}\right|^{2} .
$$

We had to compute a very large number of transitions satisfying the selection rule $\left|J_{f}-J_{i}\right|$ $\leqslant 1$, where $J_{i}$ and $J_{f}$ are the values of the angular momentum quantum number $J$ for the initial and final state, respectively. Consequently, we saved memory by organizing the calculation of the ro-vibrational eigenstates and the $S(f \leftarrow i)$-values such that at a given time, only eigenvectors for states with two consecutive $J$-values, $J$ and $J+1$, are available for the computation of $S(f \leftarrow i)$-values. This algorithm is implemented in the GPU GAIN-MPI program. ${ }^{59}$

The vibrational basis set $|V\rangle$ is obtained in TROVE using a multi-step contraction and symmetrization procedure, starting from local primitive basis set functions, each depending on one variable only (see Refs. $42-44$ and references therein). Thus, a compact representation of the vibrational basis set is obtained in a form optimized for the molecule of interest. The final vibrational basis set is represented by the eigenfunctions of the purely vibrational part of the Hamiltonian; we call these eigenfunctions the ' $J=0$ basis'.

\section{Results}

\section{Basis set convergence and empirical adjustment of the vibrational band centers}

The dimensions of the Hamiltonian matrix blocks to be diagonalized are important in determining the accuracy of the computed energies and wavefunctions for highly excited rovibrational states. Consequently it is imperative to determine empirically the smallest basis set with which the required eigenvalue accuracy (i.e., the optimum basis-set size for 'conver- 
gence') can be attained.

In TROVE, the size of the vibrational basis set is controlled by polyad number truncation. ${ }^{41-43}$ For $\mathrm{CH}_{3}$, the polyad number $P$ is defined as:

$$
P=2\left(n_{1}+n_{2}+n_{3}\right)+n_{4}+n_{5}+n_{6},
$$

where $n_{i}$ are the principal quantum numbers associated with the primitive functions $\phi_{n_{i}}\left(\xi_{i}\right)$. The primitive vibrational basis functions are products of one-dimensional basis functions $\phi_{n_{i}}\left(\xi_{i}\right)$, and only products with $P \leq P_{\max }$ are included in the primitive vibrational basis.

An even tighter level of convergence could be achieved for the vibrational term values if these were calculated with different $P_{\text {max }}$-values and the resulting progression of term values were extrapolated to the complete vibrational basis set limit. ${ }^{60}$ However, for the purpose of generating line lists this is not considered necessary. The corrections from the extrapolation will be small compared with the term-value errors caused by the imperfection of the underlying potential energy surface. Instead, we pragmatically aim for a higher accuracy by resorting to an empirical approach: The theoretical vibrational term values are replaced by the available accurate, experimentally derived vibrational band-centre values. In this manner, we are adjusting the vibrational band centers 'manually'; this empirical adjustment also shifts the rotational energy-level structure towards better agreement with experiment. We call this procedure the EBSC scheme as it can be regarded as an Empirical Basis Set Correction.

We adopt the EBSC scheme for the vibrational bands $\nu_{2}, 2 \nu_{2}, \nu_{1}, \nu_{4}^{1}$, and $\nu_{3}^{1}$, for which accurate experimental data are available, in combination with PES-2, where we have adjusted the equilibrium structure of the molecule to fit the experimentally derived pure rotational term values. The vibrational basis set was truncated at the polyad number $P_{\max }=32$. We incorporate experimental information in the EBSC scheme, and so we obviously depart from a purely ab initio approach. This is considered justified by the accuracy improvement that 
can be achieved in the computation of an extensive ro-vibrational line list.

To improve the accuracy of the predicted vibrational band-centers, a more thorough refinement of the PES would be required. However, the available accurate experimental data for the vibrationally excited states of $\mathrm{CH}_{3}$ is severely limited, and so we opted for the EBSC approach in conjunction with the $r_{\mathrm{e}}$-refinement. For all bands that are not EBSCcorrected, the predicted vibrational term values are determined to a significant extent by the ab initio data, and so their accuracy is limited. However, we have improved the prediction of the rotational structures, and that will facilitate the assignments of future experimental spectra for $\mathrm{CH}_{3}$.

In Table 1, the vibrational term values below $5000 \mathrm{~cm}^{-1}$ of the methyl radical, calculated variationally in the present work from PES-2, are compared with the available experimental data. The EBSC substitution was made in the $J>0$ TROVE calculations of the present work, in that the theoretical vibrational term values (obtained for $P_{\max }=32$ ) were replaced by the experimental values in Table 1 . This table also shows the effect of the polyad number $P_{\max }$ on the vibrational energy.

Table 2 shows a comparison of the pure rotational energies $(J \leq 5)$ of $\mathrm{CH}_{3}$ before and after refinement of $r_{\mathrm{e}}$ illustrating the importance of this step.

The vibrational transition moments are defined as

$$
\mu_{V^{\prime} V}=\sqrt{\sum_{\alpha=x, y, z}\left|\left\langle V^{\prime}\left|\bar{\mu}_{\alpha}\right| V\right\rangle\right|^{2}}
$$

where $\left|V^{\prime}\right\rangle$ and $|V\rangle$ denote $J=0$ vibrational wavefunctions and $\bar{\mu}_{\alpha}$ is the electronicallyaveraged dipole moment in the molecular frame (see the section entitled 'Dipole moment surface' above). For calculation of vibrational transition moments we used our ab initio PES-1 and truncated the vibrational basis set at polyad number $P_{\max }=32$. A number of computed transition moments for the strongest lower lying bands are listed in Table 3 where they are compared with the available experimental data. The complete list of 
Table 1: Vibrational band centers $\left(\mathrm{cm}^{-1}\right)$ of ${ }^{12} \mathrm{CH}_{3}$ from variational calculations.

\begin{tabular}{|c|c|c|c|c|c|}
\hline$\Gamma$ & State & Ref. & Obs. $^{a}$ & $P_{\max }=24^{b}$ & $P_{\max }=32^{c}$ \\
\hline \multirow[t]{11}{*}{$A_{1}^{\prime}$} & $2 \nu_{2}$ & 61 & 1288.1 & 1279.77 & 1281.24 \\
\hline & $2 \nu_{4}$ & & & 2737.63 & 2739.64 \\
\hline & $4 \nu_{2}$ & & & 2773.65 & 2776.86 \\
\hline & $\nu_{1}$ & 13 & 3004.42 & 3002.71 & 3002.76 \\
\hline & $3 \nu_{4}^{3}$ & & & 4118.59 & 4120.58 \\
\hline & $\nu_{1}+2 \nu_{2}$ & & & 4258.97 & 4260.53 \\
\hline & $6 \nu_{2}$ & & & 4391.99 & 4397.00 \\
\hline & $\nu_{3}^{1}+\nu_{4}^{1}$ & & & 4537.94 & 4538.93 \\
\hline & $4 \nu_{4}$ & & & 5371.39 & 5364.56 \\
\hline & $2 \nu_{2}+3 \nu_{4}^{3}$ & & & 5475.84 & 5480.07 \\
\hline & $4 \nu_{2}+2 \nu_{4}$ & & & 5601.91 & 5607.20 \\
\hline \multirow[t]{6}{*}{$E^{\prime}$} & $\nu_{4}^{1}$ & 62 & 1397.0 & 1385.99 & 1387.26 \\
\hline & $2 \nu_{2}+\nu_{4}^{1}$ & & & 2688.80 & 2691.61 \\
\hline & $2 \nu_{4}^{2}$ & & & 2759.77 & 2762.05 \\
\hline & $\nu_{3}^{1}$ & 58 & 3160.8 & 3158.88 & 3158.83 \\
\hline & $3 \nu_{4}^{1}$ & & & 4074.69 & 4075.46 \\
\hline & $2 \nu_{2}+2 \nu_{4}^{2}$ & & & 4087.92 & 4091.72 \\
\hline \multirow[t]{8}{*}{$A_{2}^{\prime \prime}$} & $\nu_{2}$ & 13 & 606.453 & 602.43 & 602.43 \\
\hline & $3 \nu_{2}$ & & & 2010.09 & 2010.09 \\
\hline & $\nu_{2}+2 \nu_{4}^{0}$ & & & 3372.27 & 3371.59 \\
\hline & $5 \nu_{2}$ & & & 3569.96 & 3569.95 \\
\hline & $\nu_{1}+\nu_{2}$ & & & 3596.35 & 3596.30 \\
\hline & $3 \nu_{4}^{3}$ & & & 4768.70 & 4767.06 \\
\hline & $\nu_{2}+2 \nu_{4}^{0}$ & & & 4823.32 & 4822.79 \\
\hline & $\nu_{1}+3 \nu_{2}$ & & & 4981.58 & 4981.52 \\
\hline \multirow[t]{7}{*}{$E^{\prime \prime}$} & $\nu_{2}+\nu_{4}^{1}$ & & & 2000.24 & 2002.22 \\
\hline & $\nu_{2}+2 \nu_{4}^{2}$ & & & 3388.24 & 3391.11 \\
\hline & $3 \nu_{2}+\nu_{4}^{1}$ & & & 3426.45 & 3430.06 \\
\hline & $\nu_{2}+\nu_{3}^{1}$ & & & 3736.40 & 3736.97 \\
\hline & $\nu_{2}+3 \nu_{4}^{1}$ & & & 4726.62 & 4728.62 \\
\hline & $3 \nu_{2}+2 \nu_{4}^{2}$ & & & 4835.22 & 4839.85 \\
\hline & $\nu_{1}+\nu_{2}+\nu_{4}^{1}$ & & & 4980.92 & 4983.16 \\
\hline
\end{tabular}

${ }^{a}$ Experimental values of band centers used to replace the theoretical values $P_{\max }=32$, see text.

${ }^{b}$ Computed using the $P_{\max }=24$ basis set in conjunction with PES-2. ${ }^{18}$

${ }^{c}$ Computed using the $P_{\max }=32$ basis set in conjunction with PES-2. ${ }^{18}$ 
Table 2: Theoretical rotational term values $\left(N \leq 5\right.$, in $\left.\mathbf{c m}^{-1}\right)$ of $\mathrm{CH}_{3}$, computed with TROVE using different equilibrium structure parameters.

\begin{tabular}{rrrrrr}
\hline \hline \multicolumn{3}{c}{ States } & \multicolumn{3}{c}{ Term values } \\
\hline$N$ & $K$ & $\tau_{\text {rot }}$ & Obs. & Obs.-Calc. $^{a}$ & Obs.-Calc. $^{b}$ \\
\hline 1 & 1 & 0 & 14.3189 & 0.032377 & 0.004027 \\
2 & 0 & 1 & 57.4396 & 0.112005 & -0.002023 \\
2 & 2 & 0 & 38.1186 & 0.092340 & 0.017004 \\
2 & 1 & 0 & 52.6112 & 0.106875 & 0.002511 \\
3 & 3 & 0 & 71.3965 & 0.179934 & 0.038989 \\
3 & 2 & 0 & 95.5353 & 0.203902 & 0.014649 \\
3 & 1 & 0 & 110.0032 & 0.219365 & 0.001200 \\
4 & 0 & 0 & 191.2473 & 0.375024 & -0.004034 \\
4 & 4 & 0 & 114.1491 & 0.295456 & 0.070301 \\
4 & 2 & 0 & 172.0038 & 0.353500 & 0.012772 \\
4 & 3 & 0 & 147.9203 & 0.327970 & 0.035289 \\
\hline
\end{tabular}

${ }^{a}$ Calculated using $r_{\mathrm{e}}=1.07736927 \AA$ and $\alpha_{\mathrm{e}}=120.0^{\circ}$ (PES-1, see text).

${ }^{b}$ Calculated using $r_{\mathrm{e}}=1.0762977119 \AA$ and $\alpha_{\mathrm{e}}=120.0^{\circ}$ (PES-2, see text).

theoretical transition moments is given as Supporting Information and can be also found at WWW. exomol.com.

\section{Intensity simulations}

The simulation of absorption spectra at a given temperature $T$ and within a particular wavenumber interval requires knowledge of the upper and lower-state energies and the Einstein coefficients $A(f \leftarrow i)$ [or the line strengths $S(f \leftarrow i)$; the relationship between $A(f \leftarrow i)$ and $S(f \leftarrow i)$ is described in Ref. 55] for all transitions in the chosen wavenumber range. In practice, however, the transitions with intensities below a chosen limit are discarded. The most straightforward presentation of the spectral data is a 'stick' diagram with the stick heights representing the integrated absorption coefficients from Eq. (17). We report here such simulations for the $\mathrm{CH}_{3}$ absorption bands in the wavenumber interval $600-1200 \mathrm{~cm}^{-1}$ for the out-of-plane bending mode $\nu_{2}$. The line strengths in Eq. (17) are computed from Eq. (16) with the spin statistical weights $g_{\mathrm{ns}}$ from Ref. 18. The simulations are based on 
Table 3: Band Centers $\nu_{f i}$ and Vibrational Transition Moments $\mu_{f i}$ for $\mathrm{CH}_{3} \cdot{ }^{a}$

\begin{tabular}{llllll}
\hline \multicolumn{1}{c}{ States } & & $\nu_{f i} / \mathrm{cm}^{-1}$ & Calc. $\mu_{f i} / \mathrm{D}$ & Obs. $\mu_{f i} / \mathrm{D}$ & Ref. \\
\hline$f$ & $i$ & & & & \\
\hline $2 \nu_{2}$ & $\nu_{2}$ & 678.81 & 0.25684 & $0.31(6)$ & {$[40]$} \\
$\nu_{2}$ & 0 & 602.43 & 0.20403 & $0.215(25)$ & {$[37-39]$} \\
$\nu_{3}^{1}$ & 0 & 3158.83 & 0.03999 & $0.03(27)$ & {$[63,64]$} \\
\hline$\nu_{4}^{1}$ & 0 & 1387.26 & 0.02931 & & \\
$2 \nu_{3}+\nu_{4}$ & 0 & 4529.74 & 0.02049 & & \\
$\nu_{1}+\nu_{4}^{1}$ & 0 & 4383.56 & 0.00866 & & \\
$2 \nu_{2}+\nu_{3}^{1}$ & 0 & 4396.18 & 0.00486 & & \\
$2 \nu_{3}^{2}$ & 0 & 6294.76 & 0.00462 & & \\
$\nu_{1}+\nu_{3}^{1}$ & 0 & 6076.68 & 0.00321 & & \\
$2 \nu_{4}^{2}$ & 0 & 2762.05 & 0.00313 & & \\
$\nu_{3}^{1}+2 \nu_{4}$ & 0 & 5864.94 & 0.00242 & & \\
$3 \nu_{4}^{1}$ & 0 & 4075.46 & 0.00186 & & \\
$2 \nu_{2}+\nu_{3}^{1}+\nu_{4}^{1}$ & 0 & 5789.16 & 0.00130 & & \\
$4 \nu_{2}$ & 0 & 5856.39 & 0.00116 & & \\
\hline
\end{tabular}

${ }^{a}$ The transitions originate in the vibrational ground state $(i=0)$ with the exception of the hot band $2 \nu_{2} \leftarrow \nu_{2}$. 
Figure 2: An overview of the absorption spectrum (cross sections) of $\mathrm{CH}_{3}$ at different temperatures $T=300,500,1000$ and $1500 \mathrm{~K}$ generated using our line list and the Gaussian line profile with the full-width-at-half-maximum of $1 \mathrm{~cm}^{-1}$.

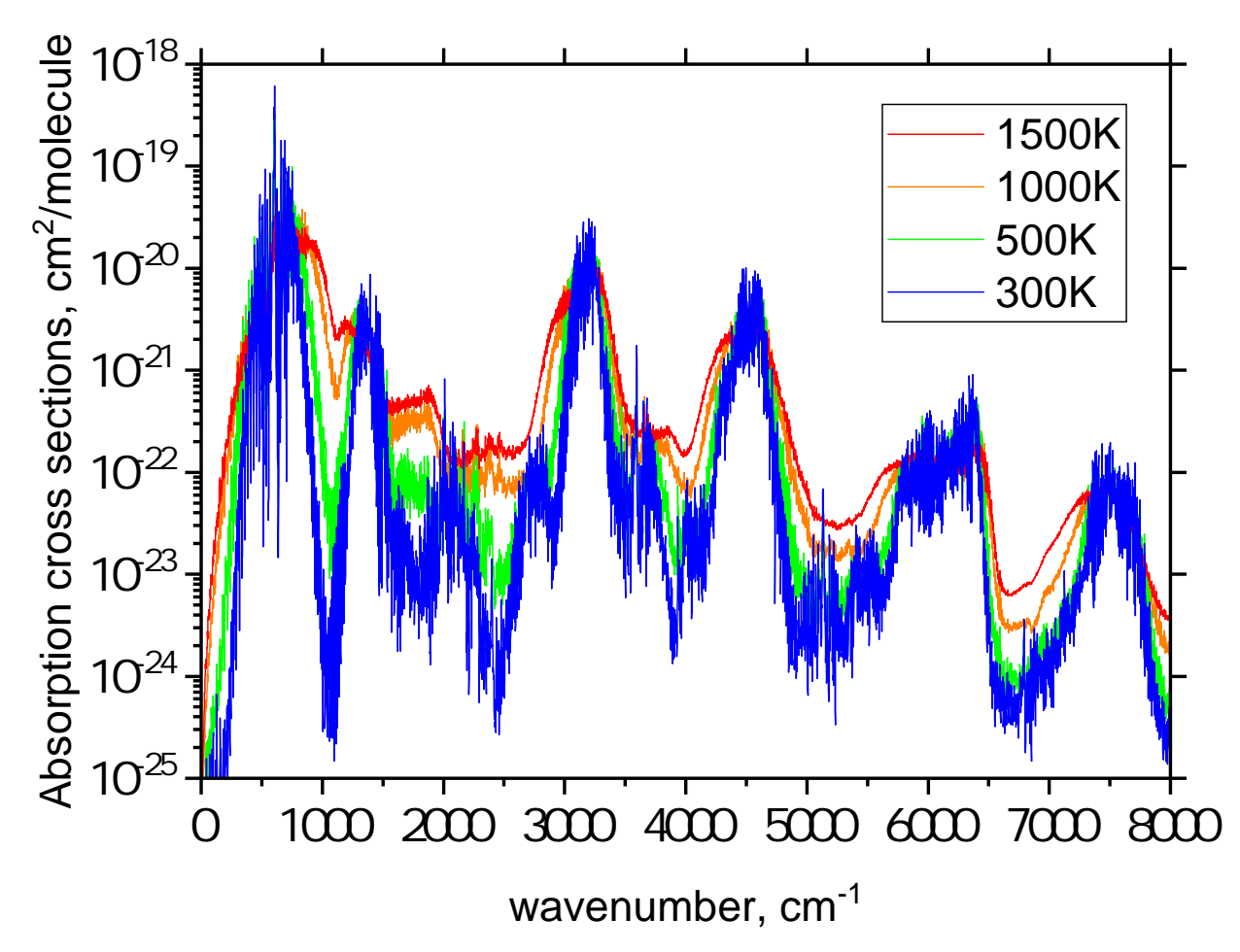

PES-2 and the computed DMS described above. The partition-function value used was $Q=$ 732.734, obtained at $300 \mathrm{~K}$ as a summation over all variational term values $(J \leq 40)$ below $36871.73 \mathrm{~cm}^{-1}$. We have computed 2,058,655,166 transitions using the GPU GAIN-MPI $\operatorname{program}^{59}$ within the various limits defined above.

Figure 2 gives an overview (log-scale) of the absorption spectrum of $\mathrm{CH}_{3}$ at different temperatures produced using the line list (log-scale) by means of the ExoCross program. ${ }^{65}$ Figure 3 shows four regions with the strongest, dipole-allowed bands $\nu_{2}, \nu_{4}, \nu_{3}$ and $\nu_{3}+\nu_{4}$.

Figure 4 shows the emission spectrum of $\mathrm{CH}_{3}$ in the wavenumber interval $600-1200 \mathrm{~cm}^{-1}$, simulated with TROVE at two different levels of theory, rovibrational and purely vibrational. The simulated spectra are compared to an experimental spectrum recorded by Hermann and Leone $^{66,67}$ (see Fig. 4 of Ref. 66). 
Figure 3: A selection of the strongest absorption bands of $\mathrm{CH}_{3}$ at $T=296 \mathrm{~K}$ generated using the line list. A Gaussian line profile with the half-width-half-maximum of $0.08 \mathrm{~cm}^{-1}$ was used in production of the cross sections shown.
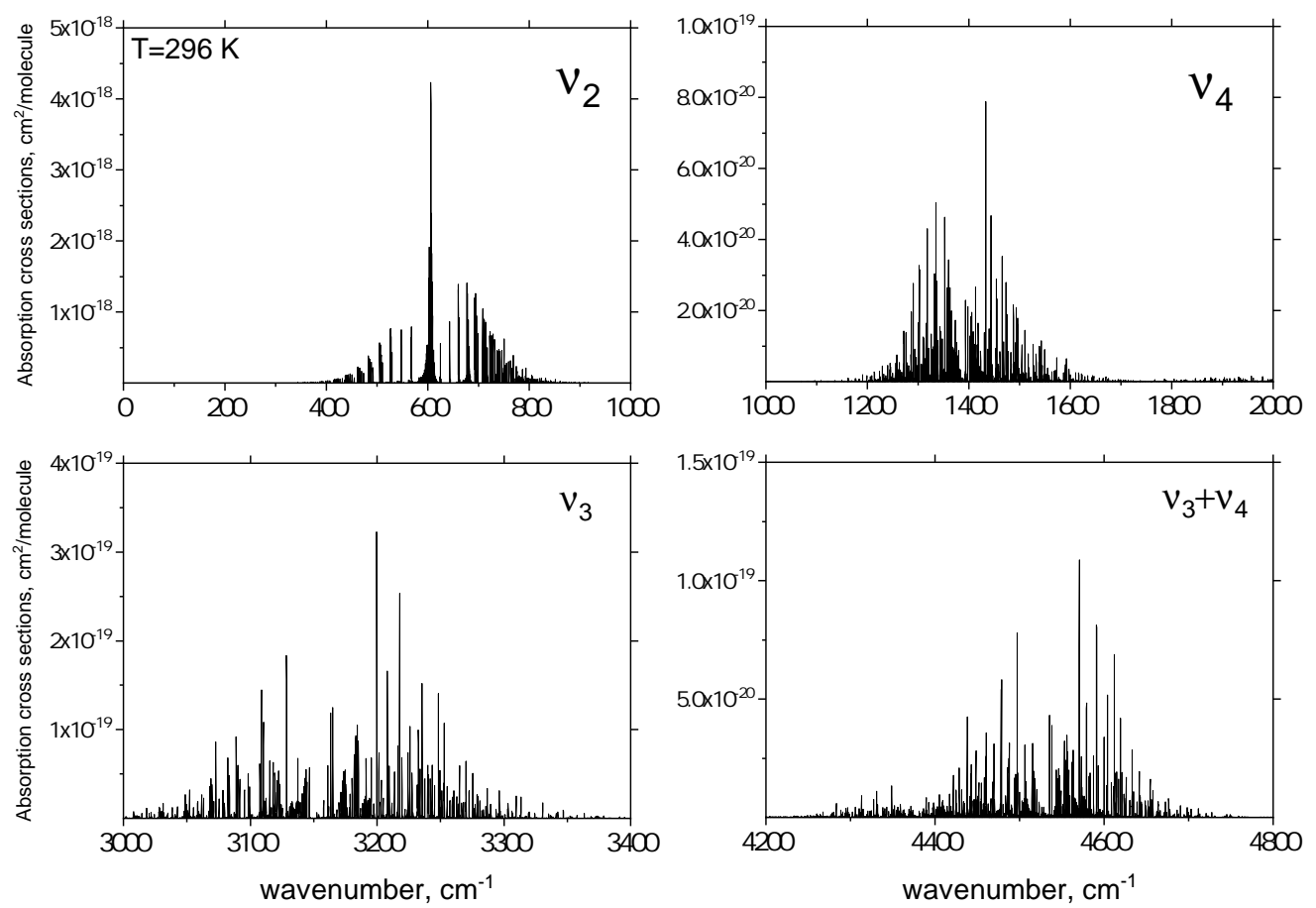

Figure 4(a) shows a simulation of the $\mathrm{CH}_{3}$ emission spectrum, computed with TROVE at a temperature of $T=300 \mathrm{~K}$, taking into account all vibrational transitions in the 600 $1200 \mathrm{~cm}^{-1}$ interval that are calculated with the $P_{\max }=32$ basis set. The simulation involves the convolution of the calculated intensities with a Gaussian line shape function with a full width at half maximum (FWHM) of $17 \mathrm{~cm}^{-1}$.

The simulation is compared with the experimental spectrum obtained by Hermann and Leone, ${ }^{66,67}$ shown in Figure 4(b).* In the experiment, the $\mathrm{CH}_{3}$ radicals were produced in a photo-fragmentation process of methyl iodide $\mathrm{CH}_{3} \mathrm{I}$. Hermann and Leone ${ }^{66,67}$ suggested that after the breaking of the $\mathrm{C}-\mathrm{I}$ bond, the $\mathrm{CH}_{3}$ radicals are predominantly produced in excited states of the out-of-plane bending mode $\nu_{2}$. The $\mathrm{CH}_{3}$ fragment of a dissociated

${ }^{*}$ Reproduced from Hermann, H. W.; Leone, S. R. The Journal of Chemical Physics 1982, 76, 4759-4765, with the permission of AIP Publishing. 
$\mathrm{CH}_{3} \mathrm{I}$ molecule will typically have a pyramidal structure close to that of the methyl group in $\mathrm{CH}_{3} \mathrm{I}$. For a $\mathrm{CH}_{3}$ radical, however, which is planar at equilibrium, such structures are associated with high excitations of the $\nu_{2}$ vibrational mode. Excitations with $v_{2} \leqslant 10$ have been observed in the emission experiment of Refs. 66,67 .

In order to simulate vibrationally very hot transitions (hotter than $1500 \mathrm{~K}$ ) corresponding to the experimental spectrum of Refs. 66 which involves out-of-plane bending states $v_{2} \nu_{2}$ with $v_{2} \leqslant 10$, we have carried out simulations employing a so-called one-band model (with the one band being the $\nu_{2}$ fundamental band here). In the one-band model, we use the $\nu_{2}$-band data from the 'parent', $300 \mathrm{~K}$ ro-vibrational line list also for the hot bands accompanying the $\nu_{2}$ band. The procedure employed is detailed in Ref. 33 and we outline it briefly here: We initially produce the $300 \mathrm{~K}, \nu_{2}$-band cross sections by means of the ExoCross program ${ }^{65}$ and convolve them with a Gaussian profile of FWHM $=17 \mathrm{~cm}^{-1}$ between 0 and $1200 \mathrm{~cm}^{-1}$, generating 1201 data points. Then the wavenumbers of the computed cross sections are shifted by $-606.4531 \mathrm{~cm}^{-1}$, positioning the band center at zero, and the cross sections are normalized. A local version of ExoCross now obtains, from the vibrational transition moments computed with TROVE, the vibrational band intensities for the hot bands $\left(v_{2}+1\right) \nu_{2}$ $\leftarrow v_{2} \nu_{2}, v_{2} \leqslant 9$. Finally, the simulated spectrum is generated by placing, for each hot band, the $\nu_{2}$-band profile at the band center $\nu_{f i}$ of the hot band in question, scaled by its vibrational band intensity. Such simulations have been carried out for temperatures $T$ of 1000,2000 , and $3000 \mathrm{~K}$, respectively, and the results are shown in Figure 4(c), where they can be compared to the experimental results ${ }^{66,67}$ in Figure 4(b).

Figure 4 shows that the 'standard' $T=300 \mathrm{~K}$ rovibrational simulation of the $\mathrm{CH}_{3}$ emission spectrum [Figure 4(a)] has little resemblance to the experimental spectrum from Ref. 66 [Figure 4(b)]. Obviously in the experiment, the $\mathrm{CH}_{3}$ molecules populate states of much higher energies than those accessed in thermal equilibrium at $T=300 \mathrm{~K}$. However, among the 'vibrational simulations' in Figure 4(c), the curve obtained for $T=2000 \mathrm{~K}$ has a very substantial similarity to the experimental curve. This confirms the suggestion by Hermann 
and Leone ${ }^{66,67}$ that dissociation of $\mathrm{CH}_{3} \mathrm{I}$ produces $\mathrm{CH}_{3}$ radicals in highly excited states of the out-of-plane bending mode $\nu_{2}$. The successful simulation of the emission spectrum of Refs. 66,67 lends credibility to the ab initio DMS of the present work; the intensities based on this DMS are in very good qualitative agreement with experiment.

Our complete $T=1500 \mathrm{~K} \mathrm{CH}_{3}$ line list is accessed via the repository www.zenodo.org, see Ref. 68. It provides transition energies, line strengths, Einstein coefficients $A(f \leftarrow i)$ and the temperature dependent partition function $Q(T)$. We expect the line list to be applicable for temperatures below $1500 \mathrm{~K}$. However, the simulated spectra will become increasingly inaccurate with increasing temperature. The line list is given in the ExoMol format ${ }^{20}$ which can be used together with the ExoCross program ${ }^{65}$ to generate spectra of $\mathrm{CH}_{3}$.

\section{Conclusion}

We report here simulations of spectra for the methyl radical, extending over a significant portion of the infrared spectral region. The positions and intensities calculated for the transitions are in excellent agreement with experiment, as demonstrated by detailed comparisons with observed room-temperature spectra.

The $\mathrm{CH}_{3}$ line list of the present work will facilitate detections of the methyl radical in space. In the present work we have generated, refined, and validated the potential energy and dipole moment surfaces required for the spectral simulations, and we have established the level of accuracy attainable in variational nuclear-motion calculations with our computational resources. We have produced a methyl radical line list consisting of 2 billion transitions between $9,127,123$ energy levels for ro-vibrational states up to $J_{\max }=40$ and energies up to $19000 \mathrm{~cm}^{-1}$. 
Figure 4: Emission spectra of $\mathrm{CH}_{3}$. (a) Rovibrational simulation assuming the $\mathrm{CH}_{3}$ radicals to be in thermal equilibrium at $T=300 \mathrm{~K}$. (b) $\mathrm{CH}_{3}\left(\nu_{2}\right)$ out-of-plane bending mode emission spectrum (dots) obtained ${ }^{66}$ after dissociation of $\mathrm{CH}_{3} \mathrm{I} \rightarrow \mathrm{CH}_{3}\left(\nu_{2}\right)+\mathrm{I}^{*}$. The continuous curve is the best fit ${ }^{66}$ involving the hot bands $\left(v_{2}+1\right) \nu_{2} \leftarrow v_{2} \nu_{2}$ with $v_{2} \leqslant 9$. The spectrum is convolved with a spectrometer slit function with a FWHM of $33 \mathrm{~cm}^{-1}$ and a $19 \mathrm{~cm}^{-1}$ bandwidth ascribed to the breadth of the $\Delta K=0$ manifold of transitions with varying $J$ values. Reproduced from Ref. 66 with the permission of AIP Publishing. (c) Vibrational simulations at temperatures $T=1000,2000$, and $3000 \mathrm{~K}$, respectively, taking into account the vibrational transitions $\left(v_{2}+1\right) \nu_{2} \leftarrow v_{2} \nu_{2}$ with $v_{2} \leqslant 9$ (see text).

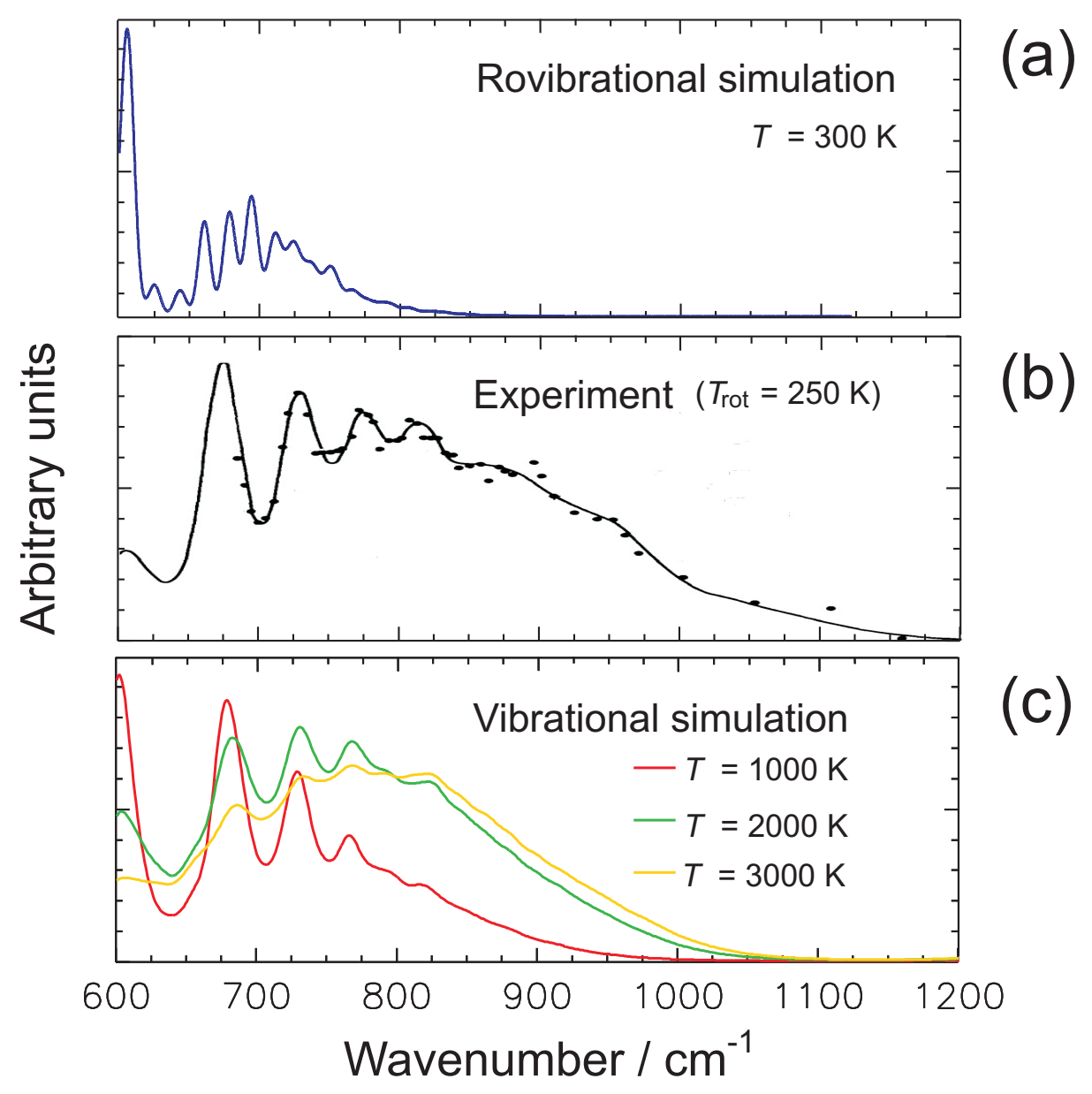




\section{Acknowledgement}

A.Y. acknowledges support from DESY (HGF IVF) and from the Cluster of Excellence 'The Hamburg Centre for Ultrafast Imaging' of the Deutsche Forschungsgemeinschaft (DFG) EXC 1074 - project ID 194651731. S.Y. is grateful for support from the UK Science and Technology Research Council (STFC) ST/R000476/1. This work made extensive use of UCL's Legion high performance (HPC) computing facilities as well as of HPC provided by DiRAC supported by STFC and BIS. It was further supported in part by grant JE 144/25-1 from the DFG.

\section{Supporting Information Available}

The Supporting Information for this work includes: (i) dipole moment parameters $\mu_{k, l, m, \ldots}^{(\Gamma)}$, (ii) potential energy parameters $f_{j k . . .}^{(s)}$; (iii) Fortran routines for calculating the dipole moment and potential energy values for a given geometry. Our complete $T=1500 \mathrm{~K} \mathrm{CH}_{3}$ line list together with the partition function can be accessed via the Zenodo repository, www .zenodo. $\operatorname{org}^{68}$ as well as at www. exomol.com. 
Figure 1 Root-mean-square (RMS) errors of the fittings to the $a b$ initio dipole moment values. The results of a series of fittings are shown. In each fitting, the data set includes the $a b$ initio points with electronic energy (relative to the potential energy minimum) $V \leqslant E$ (see text).

Figure 2 An overview of the absorption spectrum (cross sections) of $\mathrm{CH}_{3}$ at different temperatures $T=300,500,1000$ and $1500 \mathrm{~K}$ generated using our line list and the Gaussian line profile with the full-width-at-half-maximum of $1 \mathrm{~cm}^{-1}$.

Figure 3 A selection of the strongest absorption bands of $\mathrm{CH}_{3}$ at $T=296 \mathrm{~K}$ generated using the line list. A Gaussian line profile with the half-width-half-maximum of $0.08 \mathrm{~cm}^{-1}$ was used in production of the cross sections shown.

Figure 4 Emission spectra of $\mathrm{CH}_{3}$. (a) Rovibrational simulation assuming the $\mathrm{CH}_{3}$ radicals to be in thermal equilibrium at $T=300 \mathrm{~K}$. (b) $\mathrm{CH}_{3}\left(\nu_{2}\right)$ out-of-plane bending mode emission spectrum (dots) obtained ${ }^{66}$ after dissociation of $\mathrm{CH}_{3} \mathrm{I} \rightarrow \mathrm{CH}_{3}\left(\nu_{2}\right)$ $+\mathrm{I}^{*}$. The continuous curve is the best fit ${ }^{66}$ involving the hot bands $\left(v_{2}+1\right) \nu_{2} \leftarrow$ $v_{2} \nu_{2}$ with $v_{2} \leqslant 9$. The spectrum is convolved with a spectrometer slit function with a FWHM of $33 \mathrm{~cm}^{-1}$ and a $19 \mathrm{~cm}^{-1}$ bandwidth ascribed to the breadth of the $\Delta K=0$ manifold of transitions with varying $J$ values. Reproduced from Fig. 4 of Ref. 66 with the permission of AIP Publishing. (c) Vibrational simulations at temperatures $T=1000,2000$, and $3000 \mathrm{~K}$, respectively, taking into account the vibrational transitions $\left(v_{2}+1\right) \nu_{2} \leftarrow v_{2} \nu_{2}$ with $v_{2} \leqslant 9$ (see text). 


\section{References}

(1) Miller, J. A.; Kee, R. J.; Westbrook, C. K. Chemical Kinetics and Combustion Modeling. Annu. Rev. Phys. Chem. 1990, 41, 345-387.

(2) Ravishankara, R. Kinetics of Radical Reactions in the Atmospheric Oxidation of $\mathrm{CH}_{4}$. Annu. Rev. Phys. Chem. 1988, 39, 367-394.

(3) Jasinski, J. M.; Meyerson, B. S.; Scott, B. A. Mechanistic Studies of Chemical Vapor Deposition. Annu. Rev. Phys. Chem. 1987, 38, 109-140.

(4) Celii, F. G.; Butler, J. E. Diamond Chemical Vapor Deposition. Annu. Rev. Phys. Chem. 1991, 42, 643-684.

(5) Dmitriev, R. A. Z.; Dmitriev, Y. A. Detection of Free Radicals in Low-Temperature Gas-Grain Reactions of Astrophysical Interest. Astron. Astrophys. 2002, 386, 11291138.

(6) Feuchtgruber, H.; Moses, J. I.; Encrenaz, T. Detection of Methyl Radicals $\mathrm{CH}_{3}$ on Saturn. Astron. Astrophys. 1998, 44, L41-L44.

(7) Bézard, B.; Romani, P. N.; Feuchtgruber, H.; Encrenaz, T. Detection of the Methyl Radical on Neptune. Astrophys. J. 1999, 515, 868-872.

(8) Feuchtgruber, H.; Helmich, F.; van Dishoeck, E. F.; Wright, C. M. Detection of Interstellar $\mathrm{CH}_{3}$. Astrophys. J. 2000, 535, L111-L114.

(9) Settersten, T. B.; Farrow, R. L.; Gray, J. A. Coherent Infrared-Ultraviolet DoubleResonance Spectroscopy of $\mathrm{CH}_{3}$. Chem. Phys. Lett. 2003, 370, 204-210.

(10) Lefohn, A. S.; Pimentel, G. C. Infrared Spectrum of Gaseous Methyl Radical by Rapid Scan Spectroscopy. J. Chem. Phys. 1972, 57, 4028-4037. 
(11) Holt, P. L.; McCurdy, K. E.; Weisman, R. B.; Adams, J. S.; Engel, P. S. Transient CARS Spectroscopy of the $\nu_{1}$ Band of Methyl Radical. J. Chem. Phys. 1984, 81, 3349-3350.

(12) Kelly, P. B.; Westre, S. G. Resonance Raman Spectroscopy of the Methyl Radical. Chem. Phys. Lett. 1988, 151, 253-257.

(13) Triggs, N. E.; Zahedi, M.; Nibler, J. W.; DeBarber, P.; Valentini, J. J. High Resolution Study of the $\nu_{1}$ Vibration of $\mathrm{CH}_{3}$ by Coherent Raman Photofragment Spectroscopy. $J$. Chem. Phys. 1992, 96, 1822-1831.

(14) Zahedi, M.; Harrison, J. A.; Nibler, J. W. 266 nm $\mathrm{CH}_{3}$ I Photodissociation: $\mathrm{CH}_{3}$ Spectra and Population Distributions by Coherent Raman Spectroscopy. J. Chem. Phys. 1994, 100, 4043-4055.

(15) Hädrich, S.; Hefter, S.; Pfelzer, B.; Doerk, T.; Jauernik, P.; Uhlenbusch, J. Determination of the Absolute Raman Cross Section of Methyl. Chem. Phys. Lett. 1996, 256, 83-86.

(16) Westre, S. G.; Kelly, P. B. Examination of $\mathrm{CD}_{3}$ Vibrational Structure by Resonance Raman Spectroscopy. J. Chem. Phys. 1989, 90, 6977-6979.

(17) Miller, J. T.; Burton, K. A.; Weisman, R. B.; Wu, W. X.; Engel, P. S. CARS Spectroscopy of Gas Phase $\mathrm{CD}_{3}$. Chem. Phys. Lett. 1989, 158, 179-183.

(18) Adam, A. Y.; Yachmenev, A.; Yurchenko, S. N.; Jensen, P. Ro-Vibrational Averaging of the Isotropic Hyperfine Coupling Constant for the Methyl Radical. J. Chem. Phys. 2015, 143, 244306/1-7.

(19) Tennyson, J.; Yurchenko, S. N. ExoMol: Molecular Line Lists for Exoplanet and Other Atmospheres. Mon. Not. R. Astron. Soc. 2012, 425, 21-33.

(20) Tennyson, J.; Yurchenko, S. N.; Al-Refaie, A. F.; Barton, E. J.; Chubb, K. L.; Coles, P. A.; Diamantopoulou, S.; Gorman, M. N.; Hill, C.; Lam, A. Z. et al. The 
ExoMol Database: Molecular Line Lists for Exoplanet and Other Hot Atmospheres. $J$. Mol. Spectrosc. 2016, 327, 73-94.

(21) Yurchenko, S. N.; Tennyson, J. ExoMol Line Lists IV: The Rotation-Vibration Spectrum of Methane Up to 1500 K. Mon. Not. R. Astron. Soc. 2014, 440, 1649-1661.

(22) Yurchenko, S. N.; Barber, R. J.; Yachmenev, A.; Thiel, W.; Jensen, P.; Tennyson, J. A Variationally Computed $T=300 \mathrm{~K}$ Line List for $\mathrm{NH}_{3}$. J. Phys. Chem. A 2009, 113, $11845-11855$.

(23) Sousa-Silva, C.; Hesketh, N.; Yurchenko, S. N.; Hill, C.; Tennyson, J. High Temperature Partition Functions and Thermodynamic Data for Ammonia and Phosphine. Journal of Quantitative Spectroscopy \& Radiative Transfer 2014, 142, 66-74.

(24) Sousa-Silva, C.; Al-Refaie, A. F.; Tennyson, J.; Yurchenko, S. N. ExoMol Line Lists - VII. The Rotation-Vibration Spectrum of Phosphine Up to 1500 K. Mon. Not. R. Astron. Soc. 2015, 446, 2337-2347.

(25) Underwood, D. S.; Yurchenko, S. N.; Tennyson, J.; Jensen, P. Rotational Spectrum of $\mathrm{SO}_{3}$ and Theoretical Evidence for the Formation of Rotational Energy Level Clusters in its Vibrational Ground State. J. Chem. Phys. 2014, 140, 244316/1-10.

(26) Underwood, D. S.; Tennyson, J.; Yurchenko, S. N.; Clausen, S.; Fateev, A. ExoMol Line Lists XVII: A Line List for Hot $\mathrm{SO}_{3}$. Mon. Not. R. Astron. Soc. 2016, 462, 4300-4313.

(27) Al-Refaie, A. F.; Yurchenko, S. N.; Yachmenev, A.; Tennyson, J. ExoMol Line Lists VIII: A Variationally Computed Line List for Hot Formaldehyde. Mon. Not. R. Astron. Soc. 2015, 448, 1704-1714.

(28) Owens, A.; Yurchenko, S. N.; Yachmenev, A.; Tennyson, J.; Thiel, W. Accurate ab initio Vibrational Energies of Methyl Chloride. J. Chem. Phys. 2015, 142, 244306/1-10. 
(29) Owens, A.; Yurchenko, S. N.; Yachmenev, A.; Thiel, W.; Tennyson, J. ExoMol Molecular Line Lists XXII. The Rotation-Vibration Spectrum of Silane Up to 1200 K. Mon. Not. R. Astron. Soc. 2017, 471, 5025-5032.

(30) Al-Refaie, A. F.; Ovsyannikov, R. I.; Polyansky, O. L.; Yurchenko, S. N.; Tennyson, J. A Variationally Calculated Room Temperature Line-List for $\mathrm{H}_{2} \mathrm{O}_{2}$. J. Mol. Spectrosc. 2015, 318, 84-90.

(31) Al-Refaie, A. F.; Polyansky, O. L.; Ovsyannikov, R. I.; Tennyson, J.; Yurchenko, S. N. ExoMol Line Lists XV: A Hot Line List for Hydrogen Peroxide. Mon. Not. R. Astron. Soc. 2016, 461, 1012-1022.

(32) Chubb, K. L.; Yachmenev, A.; Tennyson, J.; Yurchenko, S. N. Treating Linear Molecule $\mathrm{HCCH}$ in Calculations of Rotation-Vibration Spectra. J. Chem. Phys. 2018, 149, $014101 / 1-17$.

(33) Mant, B. P.; Yachmenev, A.; Tennyson, J.; Yurchenko, S. N. ExoMol Molecular Line Lists - XXVII: Spectra of $\mathrm{C}_{2} \mathrm{H}_{4}$. Mon. Not. R. Astron. Soc. 2018, 478, 3220-3232.

(34) Gordon, I.; Rothman, L.; Hill, C.; Kochanov, R.; Tan, Y.; Bernath, P.; Birk, M.; Boudon, V.; Campargue, A.; Chance, K. et al. The HITRAN2016 Molecular Spectroscopic Database. Journal of Quantitative Spectroscopy \& Radiative Transfer 2017, 203, 3 - 69, HITRAN2016 Special Issue.

(35) Bunker, P. R.; Jensen, P. Molecular Symmetry and Spectroscopy, 2nd ed.; NRC Research Press: Ottawa, 1998; p 748.

(36) Hudgens, J. W.; DiGiuseppe, T. G.; Lin, M. C. Two Photon Resonance Enhanced Multiphoton Ionization Spectroscopy and State Assignments of the Methyl Radical. J. Chem. Phys. 1983, 79, 571-582. 
(37) Yamada, C.; Hirota, E. The Transition Dipole Moment of the $\nu_{2}$ Band of the Methyl Radical. J. Chem. Phys. 1983, 78, 669-671.

(38) Wormhoudt, J.; McCurdy, K. E. A Measurement of the Strength of the $\nu_{2}$ Band of $\mathrm{CH}_{3}$. Chem. Phys. Lett. 1989, 156, 47-50.

(39) Stancu, G. D.; Röpcke, J.; Davies, P. B. Line Strengths and Transition Dipole Moment of the $\nu_{2}$ Fundamental Band of the Methyl Radical. J. Chem. Phys. 2005, 122, $014306 / 1-11$.

(40) Stancu, G. D.; Röpcke, J.; Davies, P. B. Measurement of the Transition Dipole Moment of the First Hot Band of the $\nu_{2}$ Mode of the Methyl Radical by Diode Laser Spectroscopy. J. Phys. Chem. A 2008, 112, 6285-6288.

(41) Yurchenko, S. N.; Carvajal, M.; Jensen, P.; Lin, H.; Zheng, J.; Thiel, W. RotationVibration Motion of Pyramidal XY $\mathrm{XY}_{3}$ Molecules Described in the Eckart Frame: Theory and Application to $\mathrm{NH}_{3}$. Mol. Phys. 2005, 103, 359-378.

(42) Yurchenko, S. N.; Thiel, W.; Jensen, P. Theoretical ROVibrational Energies (TROVE): A Robust Numerical Approach to the Calculation of Rovibrational Energies for Polyatomic Molecules. J. Mol. Spectrosc. 2007, 245, 126-140.

(43) Yachmenev, A.; Yurchenko, S. N. Automatic Differentiation Method for Numerical Construction of the Rotational-Vibrational Hamiltonian as a Power Series in the Curvilinear Internal Coordinates Using the Eckart Frame. J. Chem. Phys. 2015, 143, 014105/1-16.

(44) Yurchenko, S. N.; Yachmenev, A.; Ovsyannikov, R. I. Symmetry-Adapted Rovibrational Basis Functions for Variational Nuclear Motion Calculations: TROVE Approach. J. Chem. Theory Comput. 2017, 13, 4368-4381.

(45) Adler, T. B.; Knizia, G.; Werner, H.-J. A Simple and Efficient CCSD(T)-F12 Approximation. J. Chem. Phys. 2007, 12\%, 221106/1-4. 
(46) Adler, T. B.; Werner, H.-J. Local Explicitly Correlated Coupled-Cluster Methods: Efficient Removal of the Basis Set Incompleteness and Domain Errors. J. Chem. Phys. 2009, 130, 241101/1-5.

(47) Peterson, K. A.; Adler, T. B.; Werner, H.-J. Systematically Convergent Basis Sets for Explicitly Correlated Wavefunctions: The Atoms H, He, B-Ne, and Al-Ar. J. Chem. Phys. 2008, 128, 084102/1-12.

(48) Yachmenev, A.; Yurchenko, S. N.; Ribeyre, T.; Thiel, W. High-Level ab initio Potential Energy Surfaces and Vibrational Energies of $\mathrm{H}_{2}$ CS. J. Chem. Phys. 2011, 135, 074302.

(49) Lin, H.; Thiel, W.; Yurchenko, S. N.; Carvajal, M.; Jensen, P. Vibrational Energies for $\mathrm{NH}_{3}$ Based on High Level ab initio Potential Energy Surfaces. J. Chem. Phys. 2002, 117, 11265-11276.

(50) Werner, H. J.; Knowles, P. J.; Knizia, G.; Manby, F. R.; Schütz, M. Molpro: A GeneralPurpose Quantum Chemistry Program Package. Wiley Interdisciplinary Reviews: Computational Molecular Science 2012, 2, 242-253.

(51) Watts, J. D.; Gauss, J.; Bartlett, R. J. Coupled-Cluster Methods with Noniterative Triple Excitations for Restricted Open-Shell Hartree-Fock and Other General Single Determinant Reference Functions. Energies and Analytical radients. J. Chem. Phys. 1993, 98, 8718-8733.

(52) Dunning, T. H. Gaussian Basis Sets for Use in Correlated Molecular Calculations. I. The Atoms Boron through Neon and Hydrogen. J. Chem. Phys. 1989, 90, 1007-1023.

(53) Kendall, R. A.; Dunning, Jr., T. H.; Harrison, R. J. Electron Affinities of the First-Row Atoms Revisited. Systematic Basis Sets and Wave Functions. J. Chem. Phys. 1992, 96, $6796-6806$. 
(54) Yurchenko, S. N.; Carvajal, M.; Lin, H.; Zheng, J.; Thiel, W.; Jensen, P. Dipole Moment and Rovibrational Intensities in the Electronic Ground state of $\mathrm{NH}_{3}$ : Bridging the Gap Between ab initio Theory and Spectroscopic Experiment. J. Chem. Phys. 122, $104317 / 1-14$.

(55) Yurchenko, S. N.; Thiel, W.; Carvajal, M.; Lin, H.; Jensen, P. Rotation-Vibration Motion of Pyramidal XY3 Molecules Described in the Eckart Frame: The Calculation of Intensities with Application to $\mathrm{NH}_{3}$. Adv. Quantum Chem. 2005, 48, 209-238.

(56) Bunker, P. R.; Jensen, P. The Fundamentals of Molecular Symmetry, 1st ed.; CRC Press: Boca Raton, 2004; p 358.

(57) Špirko, V.; Bunker, P. R. The Potential Function and Rotation-Vibration Energy Levels of the Methyl Radical $\mathrm{CH}_{3}$. J. Mol. Spectrosc. 1982, 95, 381-390.

(58) Davis, S.; Anderson, D. T.; Duxbury, G.; Nesbitt, D. J. Jet-Cooled Molecular Radicals in Slit Supersonic Discharges: Sub-Doppler Infrared Studies of Methyl Radical. J. Chem. Phys. 1997, 107, 5661-5675.

(59) Al-Refaie, A. F.; Yurchenko, S. N.; Tennyson, J. GPU Accelerated Intensities MPI GAIN-MPI: A New Method of Computing Einstein- $A$ Coefficients. Comput. Phys. Commun. 2017, 214, 216-224.

(60) Ovsyannikov, R. I.; Thiel, W.; Yurchenko, S. N.; Carvajal, M.; Jensen, P. Vibrational Energies of $\mathrm{PH}_{3}$ Calculated Variationally at the Complete Basis Set Limit. J. Chem. Phys. 2008, 129, 044309/1-8.

(61) Yamada, C.; Hirota, E.; Kawaguchi, K. Diode Laser Study of the $\nu_{2}$ Band of the Methyl Radical. J. Chem. Phys. 1981, 75, 5256-5264.

(62) Jacox, M. E. Vibrational and Electronic Energy Levels of Polyatomic Transient Molecules. Supplement B; American Chemical Society: New York, 2005; Vol. 1. 
(63) Bethardy, G. A.; Macdonald, R. G. Direct Measurement of the Transition Dipole Moment of the $\nu_{3}$ Asymmetric C-H Stretching Vibration of the $\mathrm{CH}_{3}$ Radical. J. Chem. Phys. 1995, 103, 2863-2872.

(64) Tanarro, I.; Sanz, M. M.; Domingo, C.; Bermejo, D.; Santos, J.; Domenech, J. L. Transition Dipole Moment of the $\nu_{3}$ Band of $\mathrm{CH}_{3}$. J. Phys. Chem. 1994, 98, 58625866.

(65) Yurchenko, S. N.; Al-Refaie, A. F.; Tennyson, J. ExoCross: A General Program for Generating Spectra from Molecular Line Lists. Astron. Astrophys. 2018, 614, A131/112.

(66) Hermann, H. W.; Leone, S. R. Photofragment Infrared Emission Spectroscopy: Vibrational Progression and Potential Parameters of the $\mathrm{CH}_{3}\left(\nu_{2}\right)$ Umbrella" Mode. J. Chem. Phys. 1982, 76, 4759-4765.

(67) Hermann, H. W.; Leone, S. R. Photofragmentation Dynamics of $\mathrm{CH}_{3} \mathrm{I}$ at 248 and 266 nm: Vibrational Distributions in the $\mathrm{CH}_{3}\left(\nu_{2}\right)$ Umbrella" Mode. J. Chem. Phys. 1982, $76,4766-4774$.

(68) Adam, A. Y.; Yachmenev, A.; Yurchenko, S. N.; Jensen, P. Supplementary Material: A Variationally Computed IR Line List for the Methyl Radical $\mathrm{CH}_{3}$. 2019; Zenodo. https://doi.org/10.5281/zenodo.2607732. 


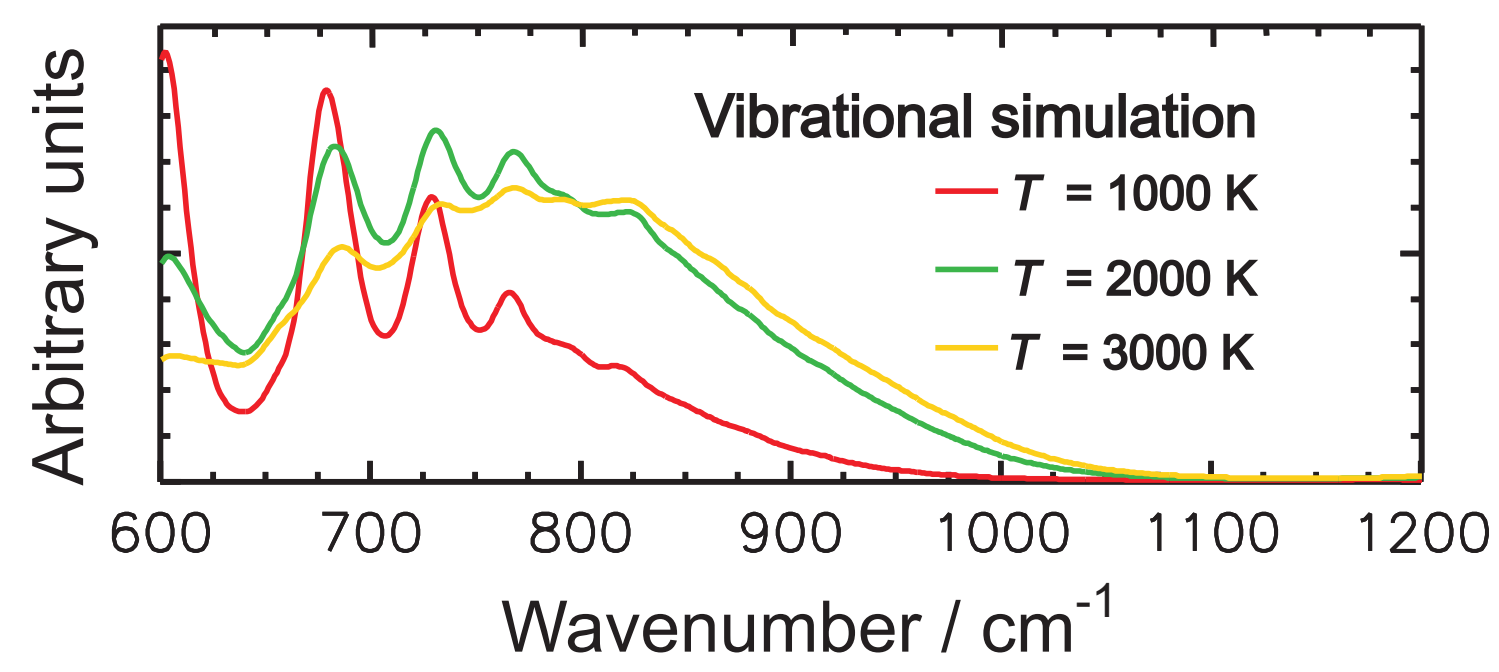

